\title{
Establishment of Trading Strategies with Value-at-Risk Models
}

\author{
Tai-Yi Yu, Chia-Lun Hsu, and Tai-Kuei Yu
}

\begin{abstract}
This study used value-at-risk ( VaR) to construct four trading strategies and simultaneously applied seven indicators to assess the suitability of the model for Taiwan Eight industries Index. To estimate the VaR, we employed three models, including the exponentially weighted moving average (EWMA), generalized autoregressive conditional heteroskedasticity (GARCH), and the Monte Carlo model, to construct suitable model parameters. Our results showed that 1) the optimum VaR model for the expansion and contraction periods of the various share indices presented significant differences in suitability and model parameters; 2) the construction of trading strategies using downside VaR can be applied to the Taiwan Eight Industries Index; 3) a comparison of the three models regarding their suitability for the eight industries index indicated that EWMA (75\%) and Monte Carlo (25\%) are applicable to a bull market, and EWMA (37.5\%), GARCH (37.5\%), and Monte Carlo (25\%) are applicable to a bear market; and 4) the proposed moving daily VaR (MDV) trading strategy shows that although the return rate of bull periods is lower than the return rate under the buy-and-hold (BH) strategy, the negative return rate of bear periods is significantly lower than the return rate under the BH strategy.
\end{abstract}

Index Terms-Monde Carlo method, EWMA, GARCH, stock index.

\section{INTRODUCTION}

Value-at-risk (VaR), a widely used risk quantification method, was defined as expected maximum loss within a particular period under normal market conditions. Four common VaR estimation methods were commonly applied, namely variance-covariance, historical simulation, Monte Carlo simulation, and extreme value theory (EVT). In this study, we used the exponentially weighted moving average (EWMA), generalized autoregressive conditional heteroskedasticity $(\mathrm{GARCH})$, and Monte Carlo simulation methods to estimate VaR values. Since development of EWMA model, studies have focused on comparisons between this model and decay factor [1]. Morgan [2] asserted that decay factors of one-day and monthly data values were 0.94 and 0.97, respectively. Besides EWMA model, GARCH is also a commonly used model for VaR estimation [3]-[6]. The Monte Carlo simulation method assumes random changes in research targets and applies random numbers to

Manuscript received February 16, 2013; revised May 25, 2013.

Tai-Yi. Yu and Chia-Lun Hsu are with the Department of Risk Management and Insurance, Ming Chuan University, Corresponding Author; 250 Zhong Shan N. Rd., Sec. 5, Taipei 111, Taiwan (e-mail: yutaiyi@gmail.com, qweszxc7410@hotmail.com).

Tai-Kuei $\mathrm{Yu}$ is with the Department of Business Administration, National Quemoy University, 1, University Rd., Jinning, Kinmen 892, Taiwan (e-mail: yutk2000@gma- il.com, yutk2012@nqu.edu.tw). simulate changes in target trends. Hendricks [7] found that, for eight countries' exchange rates from 1978 to 1995, longer observation periods increased the effectiveness of $\mathrm{VaR}$ estimations.

According to investment strategy studies, Alexander [8] proposed filter rules, using Dow Jones Industrial Average (DJIA) and S\&P stock index as research targets. Filters were set between $0.5 \%$ and $50 \%$ and investment performances were found to outperform buy-and-hold $(\mathrm{BH})$ rules regardless of whether transaction costs were considered [9]. Moreover, random walk, GARCH-M, and exponential GARCH can be considered buy-and-sell standards or momentum investing strategies [10]. The primary objectives of this study were: 1) to construct suitable trading strategies using the VaR model and apply these to an empirical study of the Taiwan Eight Industries Index; 2) to evaluate the bull and bear periods and the suitability of various models and parameters for the indices; 3) to compare and analyze the corresponding models and model parameters of bull and bear markets; and 4) to assess the suitability of applying VaR to trading strategies.

\section{RESEARCH Methodology}

For this study, the databases established by the Taiwan Economic Journal (TEJ) and the research period were selected as reference date of Taiwan's business cycles [11]. The buy-and-sell strategies employed during expansion and contraction periods differ significantly. Therefore, we adopted expansion and contraction periods from February 2005 to March 2008 and April 2008 to August 2011, respectively. Additionally, the expansion and contraction periods (respectively) contained 778 and 856 pieces of daily trading data. We focused on the Taiwan Eight Industries Index in this study, that is, the cement, food, plastic and chemical, textile, electronic, paper, construction, and financial and insurance industries. Following assumptions to accommodate research goals: 1)Investors have sufficient funds to purchase targets; 2)Investors can purchase stocks at the daily closing price when they receive the purchase signal, and can sell stocks at the daily closing price when they receive the sell signal; 3)Investors can sell all the shares owned when they receive the sell signal; 4)To prevent the possession of assets on the last day of the sample period, the last day of the sample period was set as the selling day; and 5)Transaction costs are not considered.

We used VaR with a 95\% confidence level as an analytical basis, and R software (version 2.12.1) as analytical tool. The volatility of a specific period is considered the squared return rate of that specific period and exhibits a linear relationship with volatility of the previous period (Morgan [2]; Eq. 1). 


$$
\sigma_{t}^{2}=\lambda \sigma_{t-1}^{2}+(1-\lambda) r_{t-1}^{2}
$$

where $\sigma_{t}$ denotes standard deviation (SD) of returns for $(\mathrm{t})^{\text {th }}$ period, $r_{t}$ denotes return rate for the $(t)^{\text {th }}$ period, $\mathrm{t}$ denotes period number, and $\lambda$ denotes decay factor $(0<\lambda<1)$. The range of decay factor was set as 0.90 to 0.99 , with an interval of 0.01 . The GARCH $(1,1)$ model [3] is shown in Eq. 2 .

$$
\sigma_{t}^{2}=\alpha_{o}+\alpha_{1} r_{t-1}^{2}+\beta_{1} \sigma_{t-1}^{2}
$$

where $\alpha_{i}$ and $\beta_{1}$ presented estimated parameters $(i=0,1)$. Furthermore, for Monte Carlo simulation model, this study assumes that behavior of stock price changes corresponds to geometric Brownian motion (GBM) ([12], Eq. 3).

$$
\ln \frac{S_{t+1}}{S_{t}}=\left(\mu-\frac{1}{2} \sigma\right) \delta+\sigma \sqrt{\delta} Z
$$

where $\mu$ denotes average asset return rate, $\sigma$ denotes SD of asset return rate, $\delta$ denotes time difference, and $\mathrm{Z}$ denotes random numbers generated by a standard normal distribution with a mean of 0 and an SD of 1 . Three models were employed to predict VaR values and determined quality of models with Kupiec [13] and Christoffersen [14] tests.

The volatility of a specific period is considered the squared return rate of that specific period and exhibits a linear relationship with volatility of the previous period ([2], Eq. 1). The GARCH $(1,1)$ model [3] and Monte Carlo simulation model, stock price changes corresponds to geometric Brownian motion (GBM) [12], were also applied. Three models were employed to predict $\mathrm{VaR}$ values and determined quality of models with Kupiec [13] and Christoffersen [14] tests. Four trading plocies employed in this study: 1) Daily VaR (DVaR): traders buy when return rate is lower than day downside $\mathrm{VaR}$ and sell when day return rate is higher than day upside VaR; 2) moving daily VaR (MDV): traders buy when day return rate is lower than day downside VaR and sell when three-day moving average return rate is higher than $4 \%$ (textiles 3\%); 3) five- and ten-day moving (FTDM): traders buy when five-day moving average upward penetrates ten-day moving average and sell when five-day moving average downward penetrates ten-day moving average; and 4) buy-and-hold (BH): traders buy on the first day and sell on the last day.

TABLE I: THE ARRANGEMENT OF CHANNELS

\begin{tabular}{ll}
\hline \hline Indicators & Scores \\
\hline Occurrence of negative returns & Positive denotes as 1; negative as 2 \\
\hline $\begin{array}{l}\text { Cumulative rate of return } \\
\text { Positive revenue ratios }\end{array}$ & 1-13, the best value ranked 1 \\
Return-to-risk ratio & \\
Return-to-transaction ratio $^{2}$ & \\
\hline Penetration test (Kupiec) & Optimal denotes as 1; poor as 2 \\
Continuous penetration test & \\
(Christoffersen) & \\
\hline
\end{tabular}

${ }^{1}$ The equation for this ratio is the return rate divided by the SD of the return rate. ${ }^{2}$ This indicator reflects the return rates of the unit transaction times.

We used the following seven indicators (Table I) to determine the quality of trading strategies: 1) occurrence of negative returns, 2) return rates, 3) positive revenue ratios, 4) penetration tests, 5) continuous penetration tests, 6) return-to-risk ratios, and 7) return-to-transaction ratios. The overall score is the sum of all indicator values and the lowest overall score was the optimal model.

\section{RESUltS AND Discussions}

In this study, we set the expansion period from February 2005 to March 2008 with 778 pieces of daily trading data, and similarly set the contraction period from April 2008 to August 2011 with 856 pieces of daily trading data. Changes in the daily return rates for the eight industries index during the expansion period are shown in Fig. 1.
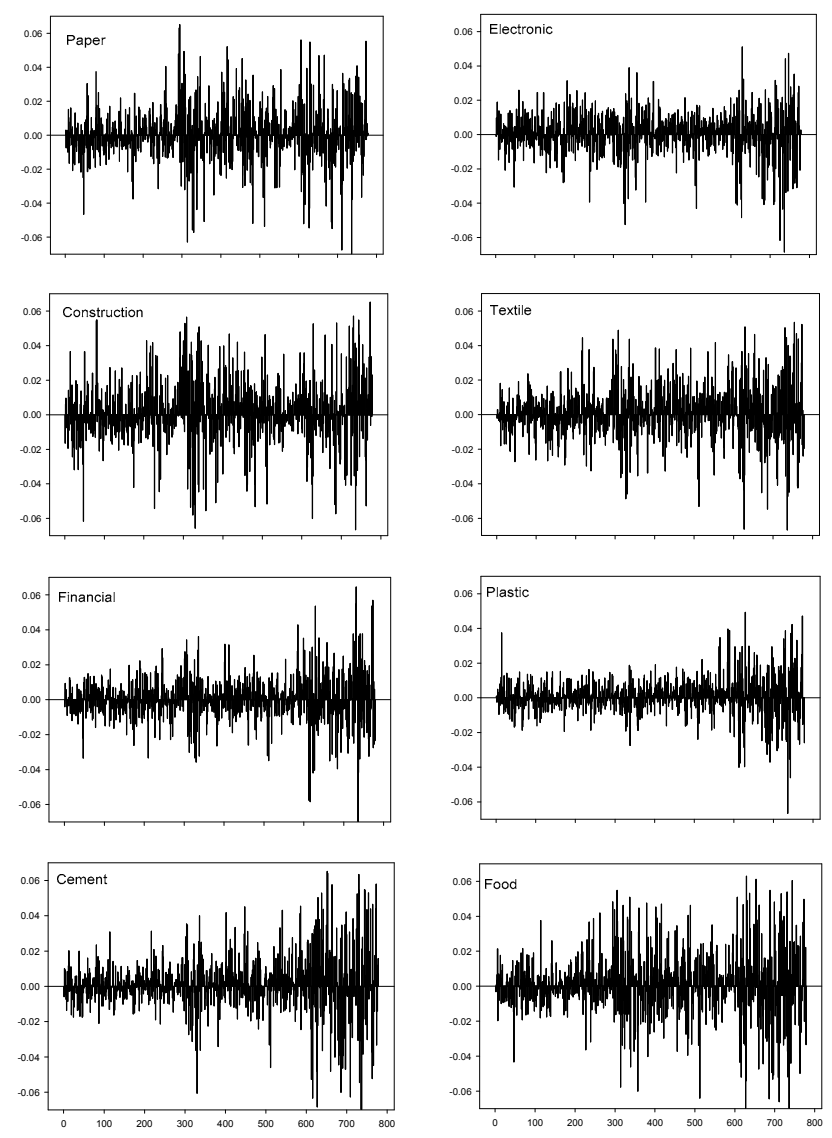

Fig. 1. Daily reward (\%) of eight industries index during expansion period.

Volatility was higher from July 2007 to February 2008 for the eight industries index. Volatility was higher from July 2007 to February 2008 for the eight industries index. Additionally, higher volatility was exhibited from May 2006 to July 2006 for the paper, construction, food, and cement industries. The results for the descriptive statistics of the daily return rates were tabulated. The top three average rates of daily returns during the expansion period were achieved by the cement $(0.12 \%)$, food $(0.12 \%)$, and construction $(0.12 \%)$ industries. The top three SDs of the daily return rates were achieved by the food $(1.96 \%)$, construction $(1.95 \%)$, and cement $(1.77 \%)$ industries. The top three average rates of the daily returns during the contraction period were achieved by the food $(0.04 \%)$, plastic and chemical $(0.01 \%)$, and textile $(-0.01 \%)$ industries. The top three industries of the daily return rates were achieved by the construction $(2.55 \%)$, financial and insurance $(2.13 \%)$, and cement $(2.11 \%)$ industries. Changes in daily return rates for eight industries 
index during the expansion period were used in this study.

We applied EWMA $(0.90<\lambda<0.99$, with an interval of 0.01), GARCH, and Monte Carlo simulation methods to simulate the VaR values. Table II shows parameters of 12 models for cement industry during expansion period. After parameters were determined, we assessed seven indicators of 12 models to identify the optimal model. Within the optimal model, seven indicators produced a total score of 12, which was lower than that of the other models; therefore, cement industry can be characterized using the EWMA model $(\lambda=0.95)$. Table 3 shows the suitable models for each industry index during the expansion and contraction periods. The Monte Carlo method can be applied to the electronic and financial and insurance industries during the expansion period, and cumulative return of the two industries was below $2 \%$. This was significantly lower than that of the other six industries. The business cycle of the financial and insurance industry is more rapid than that of other industries. The trend diagram for finance and insurance shows no significant bull trend compared with other industries; therefore, Monte Carlo simulation model (non-moving window) can be applied to finance and insurance. The remaining industries were the cement, food, plastic and chemical, textile, paper, and construction industries. The trends for these industries show significant increases. This suggests that the EWMA method is the most suitable model; however, slight differences in parameters remained.

TABLE II: MODEl PARAMETERS OF CEMENT INDUSTRY INDEX DURING EXPANSION PERIOD

\begin{tabular}{|c|c|c|c|c|c|c|c|c|c|c|c|c|}
\hline \multirow[b]{2}{*}{ Items } & \multicolumn{10}{|c|}{$\operatorname{EWMA}(\lambda)$} & \multirow{2}{*}{ GARCH } & \multirow{2}{*}{$\begin{array}{r}\text { Monde } \\
\text { Carlo }\end{array}$} \\
\hline & 0.90 & 0.91 & 0.92 & 0.93 & 0.94 & 0.95 & 0.96 & 0.97 & 0.98 & 0.99 & & \\
\hline Prediction & 518.2 & 684.4 & 730.0 & 730.0 & 720.7 & 777.7 & 514.0 & 234.5 & 332.9 & -88.1 & 217.6 & 239.9 \\
\hline Reward, \% & 23.55 & 26.32 & 27.01 & 27.04 & 26.69 & 28.80 & 17.13 & 7.33 & 10.09 & -2.59 & 5.06 & 6.66 \\
\hline Pos. Reward, \% & 77 & 81 & 81 & 81 & 78 & 78 & 70 & 66 & 70 & 59 & 63 & 67 \\
\hline Cum. Reward, \% & 6.09 & 6.92 & 7.01 & 7.01 & 6.91 & 7.31 & 4.44 & 1.79 & 2.37 & -0.56 & 1.04 & 1.24 \\
\hline Reward Range, \% & 27.88 & 40.69 & 40.69 & 40.69 & 40.69 & 40.69 & 27.05 & 25.36 & 26.62 & 36.62 & 20.45 & 20.40 \\
\hline S.D. of Revenue & 6.98 & 8.92 & 8.74 & 8.74 & 8.84 & 8.79 & 9.01 & 6.52 & 5.37 & 6.35 & 4.69 & 5.08 \\
\hline Exception numbers & 22 & 26 & 27 & 27 & 27 & 27 & 30 & 32 & 33 & 34 & 43 & 36 \\
\hline Christoffersen LR & 1 & 2 & 3 & 3 & 3 & 3 & 3 & 3 & 4 & 5 & 6 & 7 \\
\hline Buy Numbers & 22 & 26 & 27 & 27 & 27 & 27 & 30 & 32 & 33 & 34 & 43 & 36 \\
\hline Reward-to-risk,\% & 0.87 & 0.78 & 0.80 & 0.80 & 0.78 & 0.82 & 0.49 & 0.27 & 0.44 & -0.09 & 0.22 & 0.24 \\
\hline Average reward, $\%$ & 0.28 & 0.27 & 0.26 & 0.26 & 0.26 & 0.27 & 0.15 & 0.06 & 0.07 & -0.02 & 0.02 & 0.03 \\
\hline Scores Reward,\% & 1 & 1 & 1 & 1 & 1 & 1 & 1 & 1 & 1 & 2 & 1 & 1 \\
\hline Cum. Reward, \% & 6 & 4 & 2 & 2 & 5 & 1 & 7 & 9 & 8 & 13 & 11 & 10 \\
\hline Positive Reward, \% & 6 & 1 & 1 & 1 & 4 & 4 & 7 & 10 & 7 & 12 & 11 & 9 \\
\hline Kuepic LR & 1 & 1 & 1 & 1 & 1 & 1 & 1 & 2 & 2 & 2 & 2 & 2 \\
\hline Christoffersen LR & 1 & 1 & 1 & 1 & 1 & 1 & 1 & 1 & 1 & 1 & 1 & 1 \\
\hline Reward-to-risk & 1 & 5 & 3 & 3 & 5 & 2 & 7 & 9 & 8 & 13 & 11 & 10 \\
\hline Avg. Reward(\%) & 1 & 2 & 4 & 4 & 4 & 2 & 7 & 9 & 8 & 13 & 11 & 10 \\
\hline Summation & 17 & 15 & 13 & 13 & 21 & 12 & 31 & 41 & 35 & 56 & 48 & 43 \\
\hline
\end{tabular}

Changes in daily return rates for eight industries index during the expansion period were used in this study. We applied EWMA $(0.90<\lambda<0.99$, with an interval of 0.01$)$, GARCH, and Monte Carlo simulation methods to simulate the VaR values. Table 2 shows parameters of 12 models for cement industry during expansion period. After parameters were determined, we assessed seven indicators of 12 models to identify the optimal model. Within the optimal model, seven indicators produced a total score of 12 , which was lower than that of the other models; therefore, cement industry can be characterized using the EWMA model $(\lambda=0.95)$. Table 3 shows the suitable models for each industry index during the expansion and contraction periods. The Monte Carlo method can be applied to the electronic and financial and insurance industries during the expansion period, and cumulative return of the two industries was below $2 \%$. This was significantly lower than that of the other six industries. The business cycle of the financial and insurance industry is more rapid than that of other industries. The trend diagram for finance and insurance shows no significant bull trend compared with other industries; therefore, Monte Carlo simulation model (non-moving window) can be applied to finance and insurance. The remaining industries were the cement, food, plastic and chemical, textile, paper, and construction industries. The trends for these industries show significant increases. This suggests that the EWMA method is the most suitable model; however, slight differences in parameters remained.

For expansion period, cumulative return rate of MDV trading strategy was significantly higher than that of DVaR and FTDM, but lower than that of BH. When we executed MDV strategy, we could effectively delay time of share sales to increase cumulative return rates. The $\mathrm{BH}$ strategy outperformed other trading strategies during bull period; we should then avoid frequent trading during this period to obtain higher returns. The common FTDM strategies adopted in stock markets show that cumulative return rates during the expansion period are significantly lower than those under the DVaR and MDV strategies. The EWMA method is more applicable to stock markets during expansion period when trends are slowly and constantly increasing. Furthermore, volatility becomes smoother as $\lambda$ increases. The GARCH $(1,1)$ model is more suited for greater volatility; therefore, it should not be employed for expansion periods. The initial contraction period began during global financial crisis when each industry exhibited significant volatility.

Consequently, the probability of applying the GARCH $(1,1)$ model increased. The penetrability effects of the three models were superior, whereas those for continuous 
penetrability were poor. The return rates of four industries (textile, paper, construction, and financial and insurance) under the MDV strategy were lower than that under the FTDM strategy.

TABLE III: THE OPTIMAL MODELS AND REWARD (\%) OF FOUR TRADING POLICIES FOR EIGHT INDUSTRIALS INDEX

\begin{tabular}{|c|c|c|c|c|c|c|c|c|c|c|}
\hline & \multicolumn{5}{|c|}{ Expansion(\%)/Trading Policy } & \multicolumn{5}{|c|}{ Contraction (\%)/Trading Policy } \\
\hline & Optimal models & DVaR & MDV & FTDM & $\mathrm{BH}$ & Optimal models & $D V a R$ & MDV & FTDM & $\mathrm{BH}$ \\
\hline Cement & EWMA, $\lambda=0.95$ & 7.31 & 57.14 & 12.73 & 156.34 & Monde Carlo & 4.38 & 4.25 & 0.18 & $\begin{array}{r}-26.0 \\
0\end{array}$ \\
\hline Food & EWMA, $\lambda=0.90$ & 6.33 & 40.85 & 3.04 & 159.88 & $\operatorname{GARCH}(1,1)$ & 2.77 & 6.05 & 0.63 & 28.70 \\
\hline Plastic & EWMA, $\lambda=0.95$ & 2.35 & 25.74 & 1.28 & 70.28 & Monde Carlo & 1.06 & 2.41 & 0.15 & 4.93 \\
\hline Textile & EWMA, $\lambda=0.90$ & 5.10 & 30.78 & 3.86 & 91.08 & EWMA, $\lambda=0.98$ & 0.95 & -11.07 & 0.13 & $\begin{array}{r}-16.0 \\
0\end{array}$ \\
\hline Electronic & Monde Carlo & 1.15 & 10.88 & 0.28 & 41.05 & $\operatorname{GARCH}(1,1)$ & 1.35 & 8.26 & -0.97 & $\begin{array}{r}-15.6 \\
2\end{array}$ \\
\hline Paper & EWMA, $\lambda=0.90$ & 2.12 & 14.94 & 1.55 & 17.00 & $\operatorname{GARCH}(1,1)$ & 1.92 & -9.25 & 0.54 & $\begin{array}{r}-14.6 \\
4\end{array}$ \\
\hline Construction & EWMA, $\lambda=0.90$ & 2.06 & 12.60 & 1.44 & 139.93 & EWMA, $\lambda=0.96$ & -3.45 & -11.52 & 0.10 & $\begin{array}{r}-30.7 \\
7\end{array}$ \\
\hline Financial & Monde Carlo & 1.75 & 10.49 & 0.80 & 12.00 & EWMA, $\lambda=0.96$ & -0.46 & -3.21 & -1.14 & $\begin{array}{r}-25.0 \\
5\end{array}$ \\
\hline
\end{tabular}

Additionally, return rates of DVaR strategy were only lower than those of FTDM strategy for construction and financial and insurance industries. The probability of attaining return rates that exceed those provided by FTDM is greater when trading strategies are constructed with the VaR model. Aside from the food and plastic industries, the MDV return rate was higher than that for $\mathrm{BH}$. Moreover, the Monte Carlo simulation model can be applied to cement and plastic and chemical industries for contraction period. Additionally, GARCH $(1,1)$ model could detect rapid volatility and fulfilled conditions of rapid stock index decline during contraction periods. Therefore, this model is applicable for the food, electronic, and paper industries during contraction.

\section{CONCLUSION}

In this study, we constructed suitable trading strategies by applying the EWMA, GARCH, and Monte Carlo methods for simulating $\mathrm{VaR}$ values to the Taiwan Eight Industries Index. We employed seven indicators to determine the optimum

Simulating models. Although each VaR model cannot be simultaneously applied to the eight industries index during the bull and bear periods, the results still indicate that VaR-constructed trading strategies have a certain degree of applicability during the bull and bear periods.

The optimal model for electronic and financial and insurance industries during the bull period is the Monte Carlo simulation model, which indicates that the degree of volatility for the two industries is not time-related. The EWMA model was applicable to the remaining six industries. This implies greater correlations between volatility and time. Additionally, volatility was more stable and also corresponded to the slow and long-term increase trends observed during the bull period. By contrast, the Monte Carlo model was applicable to the cement and plastic and chemical industries during the bear period, and the EWMA and GARCH models were applicable to the remaining industries. This shows that unexpected and rapid decreases in share prices occurred during the bear period; however, most of the volatility degree could still be modeled and simulated. Simulations during the bear period, as opposed to the bull period, produced better results using the GARCH model because the model can detect rapid volatility. Therefore, the volatility of the stock market during the bull period was lower than that during the bear period.

Based on the obtained maximum cumulative return rates, the BH strategy should be employed during the bull period, whereas the MDV strategy should be applied when the stock market experiences a bear period. Although the MDV strategy produces negative returns for the textile, paper, construction, and financial and insurance industries, it can prevent substantial losses through the advanced selling of related shares with the approach of a bear market. Based on conservative and moderate conditions and considerations, we recommend applying the MDV strategy. Although the cumulative return rates during the bull period for this strategy are lower than those of the BH strategy, the negative return rates during the bear period are substantially lower than those of the BH strategy. The VaR-constructed trading strategies can better control downside VaR compared to the $\mathrm{BH}$ strategy. Furthermore, the return rates of the MDV strategy are higher than the return rates of the FDTM strategy.

In this study, we excluded transaction costs to simplify the research process and sold all owned shares when we received the selling signal or a selling point appeared. Therefore, we provide the following recommendations for future studies: Transaction costs should be included to fit real life situations and downside VaR should be controlled through stop-loss mechanisms, and the method of share sales should be emphasized to consider the effect that selling ratios have on cumulative return rates.

\section{REFERENCES}

[1] Y. Y. Chuang and T. H. Tsai, "Searching EWMA optimal Lambda in Taiwan financial market," Money Watching \& Credit Rating, vol. 28, pp. 133-162, 2001.

[2] J. P. Morgan, Risk Metrics Technical Document, 4th ed., Morgan Guaranty Trust Company, 1996. 
[3] T. Bollerslev, "Generalized autoregressive conditional heteroskedasticity," Journal of Econometrics, vol. 31, pp. 307-327, 1986.

[4] P. R. Hansen and A. Lunde, "A forecast comparison of volatility models: Does anything beat a GARCH (1. 1)?" Journal of Applied Econometrics, vol. 20, pp. 873-889, 2005.

[5] F. Piotr and O. Witold, "Nonparametric Verification of GARCH-Class Models for Selected Polish Exchange Rates and Stock Indices," Czech Journal of Economics and Finance, vol. 62, pp. 430-449, 2012.

[6] A. Antoniou, H. Y. T. Lam, and K. Paudyal, "Profitability of momentum strategies in international markets: The role of business cycle variables and behavioural biases," Journal of Banking \& Finance, vol. 31, pp. 955-972, 2007.

[7] D. Hendricks, "Evaluation of Value-at-Risk models using historical data," Economic Policy Review, vol. 2, pp. 39-69, 1996.

[8] S. S. Alexander, "Price Movement in Speculative Markets: Trends or Random Walks," Industrial Management Review, vol. 2, pp. 7-26, 1961.

[9] R. J. Sweeney, "Some new filter rule tests: methods and results," Journal of Financial and Quantitative Analysis, vol. 23, pp. 285-300, 1998.

[10] S. Rachev, T. Jasic, S. Stoyanov, and F. J. Fabozzi, "Momentum strategies based on reward-risk stock selection criteria," Journal of Banking \& Finance, vol. 31, pp. 2325-2346, 2007.

[11] Council for Economic Planning and Development. Taiwan. (2010). [Online]. Available: http://www. cepd.gov.tw/m1.aspx? $\mathrm{sNo}=0000161$

[12] P. A. Samuelson, "Proof that property anticipated prices fluctuate randomly," Industrial Management Review, vol. 6, pp. 41-49, 1965.

[13] P. H. Kupiec, "Techniques for verifying the accuracy of risk measurement models," Journal of Derivatives, vol. 3, pp. 73-84, 1995.
[14] P. Christoffersen, "Evaluating interval forecasts," International Economic Review, vol. 39, pp. 841-862, 1998.

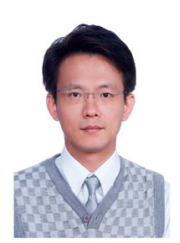

Tai-Yi Yu is living in Ilan, Taiwan, who was born in 1968. He is a Ph. D. from Graduate Institute of Environmental Engineering, National Taiwan University, Taipei, Taiwan. He also as a Assistant Professor, Department of Risk Management and Insurance, Ming Chuan University, 250. Research interests: Risk Analysis, Multivariate Analysis, Risk Assessment.

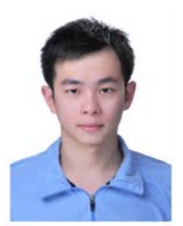

Chia-Lun Hsu is living in Kaohsiung, Taiwan, who was born in 1988. He is a MBA at the Department of Risk Management and Insurance, Ming Chuan University, Taipei, Taiwan. Research interests: Risk Analysis, Finance, Actuary.

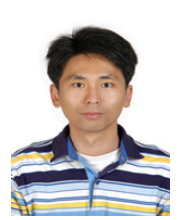

e-Commerce.
Tai-Kuei Yu is living in ChiaYi, Taiwan, who was born in 1970. Now, he is a Ph. D. at the Department of Business Administration, National Yunlin University of Science and Technology, Yunlin, Taiwan. He is the Department of Business Administration, National Quemoy University, 1, University Rd., Jinning, Kinmen 892, Taiwan. Research interests: Multivariate Analysis, Information Management, 